\title{
Abstract: Multi-camera, Multi-person, and Real-time Fall Detection using Long Short Term Memory
}

\author{
Christian Heinrich ${ }^{1}$, Samad Koita ${ }^{2}$, Mohammad Taufeeque ${ }^{2}$, Nicolai Spicher ${ }^{1}$, \\ Thomas M. Deserno ${ }^{1}$ \\ ${ }^{1}$ Peter L. Reichertz Institute for Medical Informatics of TU Braunschweig and \\ Hannover Medical School, Germany \\ ${ }^{2}$ Indian Institute of Technology Bombay, India \\ christian.heinrich@plri.de
}

Falls occurring at home are a high risk for elderly living alone. Several sensorbased methods for detecting falls exist and - in majority - use wearables or ambient sensors. Video-based fall detection is emerging. However, the restricted view of a single camera, distinguishing and tracking of persons, as well as high false-positive rates pose limitations. We have proposed the following [1]: We augment the human pose estimation algorithm openpifpaf for fall detection by adding multi-camera and multi-person tracking support. For each person, five temporal and spatial features are extracted and processed by a long short-term memory (LSTM) network, classifying each frame as a fall or no fall event. We use the UP-fall detection dataset for evaluation and achieve a F1-score of $92.5 \%$. Still, we observe a trend of false-positives, which might result from the imbalance in the data (36\% videos showing falls) with respect to their rare occurrences in real life. For improvement, we will acquire a multi-camera dataset in our smart home environment that represents falls and everyday activities.
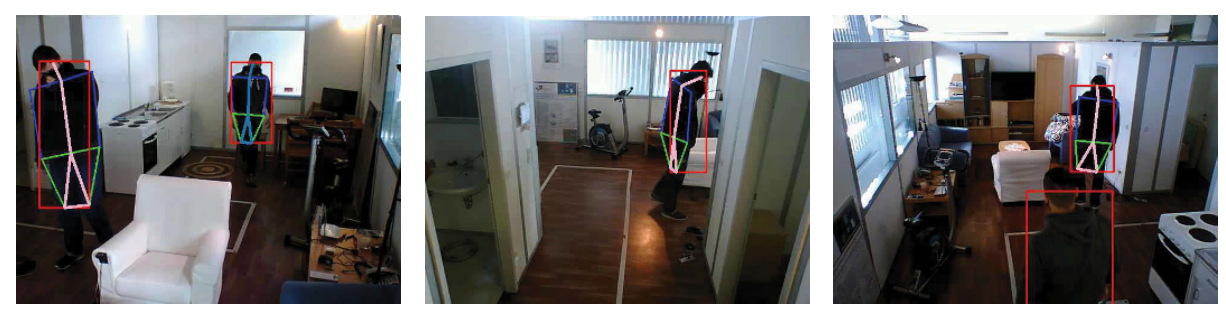

Fig. 1. Initial results acquired in the smart home lab from three cameras capturing two persons. Each person is surrounded by a red bounding box and pose key points are visualized by colored lines. Right panel: The view is restricted which underlines the need for multi-camera support. Faces are masked due to the COVID-19 pandemic.

\section{References}

1. Taufeeque M, Koita S, Spicher N, et al. Multi-camera, multi-person, and real-time fall detection using long short term memory. Proc SPIE. 2021;Accepted. 